\title{
Immature Neurons From CNS Stem Cells Proliferate in Response to Platelet-Derived Growth Factor
}

\author{
Anna Erlandsson, Maria Enarsson, and Karin Forsberg-Nilsson \\ Department of Genetics and Pathology, Rudbeck Laboratory, Uppsala University, SE-751 85 Uppsala, Sweden
}

Identifying external signals involved in the regulation of neural stem cell proliferation and differentiation is fundamental to the understanding of CNS development. In this study we show that platelet-derived growth factor (PDGF) can act as a mitogen for neural precursor cells. Multipotent stem cells from developing CNS can be maintained in a proliferative state under serum-free conditions in the presence of fibroblast growth factor-2 (FGF2) and induced to differentiate into neurons, astrocytes, and oligodendrocytes on withdrawal of the mitogen. PDGF has been suggested to play a role during the differentiation into neurons. We have investigated the effect of PDGF on cultured stem cells from embryonic rat cortex. The PDGF $\alpha$-receptor is constantly

Neurons and glia in the mature CNS originate from precursor cells in the ventricular zone of the fetal brain and spinal cord (Williams et al., 1991). The division of the precursor cells takes place close to the lumen of the neuroepithelium, after which the postmitotic progeny migrate from the ventricular zone to assume their final positions (Rakic, 1972, 1990; Hatten, 1990).

Many reports describe the establishment of in vitro culture of precursor cells from the embryonic rat or mouse brain (for review, see Gage, 1998; Temple and Alvarez-Buylla, 1999; Vescovi and Snyder, 1999). Neuroepithelial stem cells can be expanded in fibroblast growth factor-2 (FGF2)-containing serumfree medium and induced to differentiate to neurons, astrocytes, or oligodendrocytes by the withdrawal of mitogen (for review, see McKay, 1997).

Identification of external signals involved in the regulation of neural stem cell proliferation and differentiation may allow for improved transplantation methods and augment the treatment of neurodegenerative disorders. Several investigators report that in cell cultures of neuroepithelial progenitor cells, exogenously added growth factors can potentiate survival, induce proliferation, or facilitate commitment or differentiation into mature phenotypes. Besides the mitogenic effect of FGF2, it is well documented that ciliary neurotrophic factor (CNTF) acts to direct multipotent stem cells to an astrocytic fate (Johe et al., 1996; Bonni et al., 1997; Rajan and McKay, 1998). The thyroid hormone, triiodothyronine (T3), has been shown to influence oligodendrocyte differentiation (Johe et al., 1996). Two previous re-

\footnotetext{
Received June 12, 2000; revised Feb. 12, 2001; accepted Feb. 23, 2001.

This study was supported by grants from the Swedish Cancer Foundation, the Children Cancer Foundation of Sweden, Magnus Bergwall's Foundation, Wiberg's Foundation, and Selander's Foundation. We thank M. Lindström for excellent technical assistance.

Correspondence should be addressed to Karin Forsberg-Nilsson, Department of Genetics and Pathology, Rudbeck Laboratory, Uppsala University, SE-751 85 Uppsala, Sweden. E-mail: karin.nilsson@genpat.uu.se.

Copyright (C) 2001 Society for Neuroscience 0270-6474/01/213483-09\$15.00/0
}

expressed during differentiation of neural stem cells but is phosphorylated only after PDGF-AA treatment. In contrast, the PDGF $\beta$-receptor is hardly detectable in uncommitted cells, but its expression increases during differentiation. We show that PDGF stimulation leads to c-fos induction, 5'-bromo2 'deoxyuridine incorporation, and an increase in the number of immature cells stained with antibodies to neuronal markers. Our findings suggest that PDGF acts as a mitogen in the early phase of stem cell differentiation to expand the pool of immature neurons.

Key words: PDGF; neural stem cells; differentiation; neurogenesis; rat; cortex; development ports (Johe et al., 1996; Williams et al., 1997) indicate that PDGF can enhance neuronal differentiation. The possibility that PDGF acts as a mitogen for immature neurons has been discussed but not addressed experimentally.

Both PDGF ligands are present within the developing cortex at early embryonic ages. Hutchins and Jefferson (1992) detected PDGF-A-positive cells throughout the ventricular zone at mouse embryonic day (E) 11.5, which is $2 \mathrm{~d}$ after neural tube closure and before the differentiation of most glial cells in all areas of the nervous system. Some PDGF-B-reactive cells can also be found in the subventricular zone of E14 rat embryos, which is when rapid cellular proliferation and migration occur (Sasahara et al., 1992).

In the present study we aimed at clarifying the effect of PDGF on cortical neural stem cells in culture. We have found that PDGF-AA treatment increases 5'-bromo-2'deoxyuridine bromodeoxyuridine (BrdU) incorporation, and as a consequence it increases total cell number. PDGF-AA was further shown to delay the differentiation of microtubule-associated protein 2 (MAP2)/TuJ1-positive neural precursor cells, possibly by retaining committed but immature neurons in a proliferative state. Double staining with BrdU antibodies showed that PDGF markedly increased the proportion of proliferating cells that expressed MAP2. These results suggest that PDGF acts to expand a pool of immature neurons.

\section{MATERIALS AND METHODS}

Cell culture. Embryonic cortex was dissected in HBSS (Life Technologies, Paisley, Scotland) from timed-pregnant Sprague Dawley rats (B\&K, Sollentuna, Sweden) on E15 (E1 was defined as the day of copulatory plug). The tissue was gently triturated, and meninges and larger cell clumps were allowed to sediment for $10 \mathrm{~min}$, after which the cell suspension was centrifuged. The pellet was resuspended in N2-medium (Bottenstein and Sato, 1979) with $10 \mathrm{ng} / \mathrm{ml} \mathrm{FGF2} \mathrm{(Pepro} \mathrm{Technologies,}$ London, England) and plated at a density of $1 \times 10^{-6}$ cells per $10 \mathrm{~cm}$ tissue culture dish that was precoated with poly-L-ornithine (Sigma, St. Louis, MO) and fibronectin (Life Technologies). Fresh FGF2 was added daily, and the medium was changed every other day. When subconfluent, 
the cells were passaged by scraping with a cell lifter (Costar, Cambridge, MA). After the cells were gently triturated in HBSS, the cell suspension was centrifuged, and the pellet was resuspended in N2-medium and plated. The cells were used for experiments $2-6 \mathrm{~d}$ after the first passage. The concentrations of PDGF-AA (Pepro Technologies) used in the experiments were $10 \mathrm{ng} / \mathrm{ml}$ for continuous treatment, $20 \mathrm{ng} / \mathrm{ml}$ for treatment with a single dose, and $100 \mathrm{ng} / \mathrm{ml}$ for shorter exposures (c-fos and protein kinase $\mathrm{B}$ (PKB)/c-Akt experiments).

Immunocytochemistry. Cells were fixed in ice-cold acid ethanol $(90 \%$ ethanol, $5 \%$ acetic acid). Endogenous peroxidase was quenched by $0.3 \%$ $\mathrm{H}_{2} \mathrm{O}_{2}$ in methanol for 20 min followed by washing with PBS and permeabilization and blocking in $0.1 \%$ Triton X-100 with 5\% normal goat serum (Dako A/S, Glostrup, Denmark) in PBS for $30 \mathrm{~min}$. The cells were incubated for 1-4 hr with primary antibody, washed in PBS, and incubated with biotinylated goat anti-mouse immunoglobulin (Kirkegaard and Perry Laboratories, Gaithersburg, MD) for $30 \mathrm{~min}-1 \mathrm{hr}$. After washing with PBS, the reaction was developed with diaminobenzidine (DAB SigmaFast, Sigma). Incorporation of BrdU was detected using anti-BrdU antibodies (Amersham Pharmacia Biotech, Uppsala, Sweden). Cells were pulsed with $10 \mathrm{~mm}$ BrdU (Amersham Pharmacia Biotech) for $14 \mathrm{hr}$ before fixation. Stained cells were counted, and the ratio of BrdU-positive cells to total cell number per field at $200 \times$ magnification was calculated. Seven parallel fields were counted for each time point, and each time point was performed in duplicate. For the double staining of MAP2 and BrdU, a cell proliferation kit, including a nickel-enhanced peroxidase reaction (Amersham Pharmacia Biotech), was used for detection of BrdU-positive cells together with the regular peroxidase reaction for MAP2 as described above. Duplicate cultures were stained for MAP2, and 10 defined fields of each culture dish were photographed, using a computerized microscope (Axiovert 100TV, Zeiss). After BrdU staining, the same fields were photographed again. Cells counts were determined from photographs of stained cells. Monoclonal anti-MAP2 antibody (clone HM2, Sigma), dilution 1:200, and monoclonal anti-Tuj1 antibody (BabCO, Nordic Biosite AB, Täby, Sweden), dilution 1:50, were used. A polyclonal antibody to nestin was a kind gift from Dr. R. McKay (National Institutes of Health, Bethesda, MD).

Deoxynucleotidyl transferase-mediated biotinylated UTP nick end labeling assay. Cells were grown on poly-L-ornathine and fibronectin precoated glass coverslips. After fixation in $2 \%$ paraformaldehyde, $\mathrm{pH} 7.4$, the cells were stained using a terminal deoxynucleotidyl transferase-mediated biotinylated UTP nick end labeling (TUNEL) kit (Roche Diagnostics, Mannheim, Germany). The proportion of cells going through apoptosis was counted in 10 parallel fields, using a fluorescence microscope. Each time point was performed in duplicate.

Cell counting. Cell counting was performed using a Coulter Z1 cell counter (Coulter Electronics, Herpendon, UK). The cells were removed from the dishes by scraping and were then carefully dissociated and counted. Each experiment was performed in triplicate.

Northern blot analysis. Total RNA was isolated using the $\mathrm{LiCl} / \mathrm{urea}$ method (Auffrey and Rougeon, 1980). For Northern electrophoresis, 12 $\mu \mathrm{g}$ of RNA per slot was loaded to a $1 \%$ agarose gel with $2.2 \mathrm{M}$ formaldehyde. The RNA was transferred to Duralon-UV membranes (Stratagene, La Jolla, CA) and fixed by baking at $80^{\circ} \mathrm{C}$ under vacuum. ${ }^{32} \mathrm{P}-$ labeled probes were prepared using a kit for random priming (Megaprime DNA labeling systems, Amersham Pharmacia Biotech), and hybridization was performed under high stringency conditions $(50 \%$ formamide) at $42^{\circ} \mathrm{C}$ using QuickHyb solution (Stratagene). The intactness and total amount of RNA were checked by hybridization to a glyceraldehyde phosphate dehydrogenase (GAPDH) probe. After washing at $55-60^{\circ} \mathrm{C}$, the filters were exposed to Phosphor Imager screens (Fuji, Tokyo, Japan) for 12-14 hr and analyzed in a Phosphor Imager (Fuji). Normalization of PDGF receptor RNA levels was obtained by scanning the hybridization signal, and the ratio of receptor signal to GAPDH signal was calculated. A cDNA for GAPDH was a gift from Dr. R. Wu (Cornell University, Ithaca, NY), and murine cDNA for c-fos was kindly provided by Dr. R. Wallrich (EMBL, Heidelberg, Germany).

Western blot analysis: total lysate. Cells were lysed in boiling $2 \times$ Laemmli sample buffer (4\% SDS, 20\% glycerol, Tris-HCl, pH 6.8) and sonicated, and the cell debris was removed by centrifugation. Protein samples were boiled with $5 \%$ betamercaptoethanol and loaded to the wells of a $8-16 \%$ gradient Tris-glycine gel (Novex). For immunoblotting, the samples were electrically transferred onto a nitrocellulose membrane (Hybond ECL Nitrocellulose membrane, Amersham Pharmacia Biotech) according to the protocol provided by the manufacturer. Nonspecific protein binding to the filter was blocked by incubation in Tris-buffered
saline/Tween (TBS-T: $20 \mathrm{~mm}$ Tris base, $\mathrm{pH}$ 7.6, $137 \mathrm{~mm} \mathrm{NaCl}, 0.2 \%$ Tween-20) containing 5\% BSA for $1 \mathrm{hr}$ at room temperature, followed by incubation with primary antibody overnight at $4^{\circ} \mathrm{C}$. After washing with TBS-T, the membrane was incubated with peroxidase-conjugated secondary antibody (Amersham Pharmacia Biotech), dilution 1:5000, for 1 $\mathrm{hr}$ at room temperature. After the filter was washed in TBS-T, the blot was developed using an enhanced chemiluminescence (ECL) system (Amersham Pharmacia Biotech). Before rehybridization of the filters, the membrane was dehybridized (20\% SDS, Tris-HCl, pH 6.8, $340 \mu$ l betamercaptoethanol) at $55^{\circ} \mathrm{C}$ for $30 \mathrm{~min}$ and washed in TBS-T. Primary antibodies were anti-PKB/c-Akt antibody (Akt1 \#06-558, Upstate Biotech) and antiphosphorylated-PKB/c-Akt antibody (Ser-473-PhosphoAkt \#9271S, New England Biolabs), diluted 1:1000.

Western blot analysis: immunoprecipitation. Cell pellets were solubilized in lysis buffer (1\% Triton X-100, $20 \mathrm{~mm}$ Tris-HCl, pH 7.5, $150 \mathrm{~mm}$ $\mathrm{NaCl}, 2 \mathrm{~mm}$ EDTA, $1 \%$ aprotinin, $2 \mathrm{~mm}$ phenylmethylsulfonyl fluoride, $250 \mu \mathrm{M}$ orthovanadate) on ice for $5 \mathrm{~min}$, after which cell debris was removed by centrifugation. Immunoprecipitation was performed using $150 \mu \mathrm{g}$ protein. Lysates were incubated with $10 \mu \mathrm{l}$ anti-PDGF $\alpha$-receptor antibody (anti-PDGF-R $\alpha$ antibody), R7, for $90 \mathrm{~min}$ at $4^{\circ} \mathrm{C}$ and another 45 min on ice after the addition of $50 \mu \mathrm{l}$ of protein-A-Sepharose beads (Amersham Pharmacia Biotech). The supernatant was then incubated with $10 \mu \mathrm{l}$ of anti-PDGF $\beta$-receptor antibody (anti-PDGF-R $\beta$ antibody), R33, and new protein-A-Sepharose beads. The R7 and R33 antibodies were kind gifts from Dr. C.-H. Heldin (Ludwig Institute for Cancer Research, Uppsala, Sweden). The protein-A-Sepharose was washed three times with lysis buffer and once with ice-cold PBS. To eluate the protein, the samples were incubated with $25 \mu \mathrm{l}$ of SDS-sample buffer (Novex, San Diego, CA) with $2 \%$ betamercaptoethanol at $95^{\circ} \mathrm{C}$ for $5 \mathrm{~min}$. The supernatant was loaded to the wells of a $4-12 \%$ gradient Trisglycine gel (Novex). Immunoblotting was performed as above but with a different filter (Immobilon transfer membrane; Millipore, Bedford, MA). Primary antibodies were anti-phosphotyrosine antibody, PY20 (diluted 1:1250; Transduction Laboratories, Exeter, UK), anti-PDGF $\alpha$-receptor, R7 (diluted 1:500), or anti-PDGF $\beta$-receptor, M20 (diluted 1:500; Santa Cruz Biotechnology, Santa Cruz, CA).

Statistical analysis. For statistical analysis we used ANOVA followed by Fisher's multiple comparison. Values presented are the means \pm SEM.

\section{RESULTS}

\section{Expression of PDGF receptors during stem cell differentiation}

The cell culture system used in this study has been described in detail elsewhere (Johe et al., 1996). Briefly, multipotential neural stem cells can be maintained in the presence of FGF2 and induced to differentiate on withdrawal of the mitogen to form neurons, astrocytes, and oligodendrocytes. During the first few days after FGF2 withdrawal, addition of polypeptide growth factors, cytokines, or hormones can change the proportion of cell lineages that are generated. PDGF has been suggested to augment neuronal differentiation in two studies (Johe et al., 1996; Williams et al., 1997). In this study we aimed to define the action of PDGF on neuronal stem cells isolated from embryonic rat cortex. We have shown previously that these cells express PDGF $\alpha$-receptor RNA and can respond to PDGF by chemotaxis (Forsberg-Nilsson et al., 1998).

Western blot analysis was used to investigate the expression and phosphorylation of PDGF receptors in cultured neuroepithelial cells during differentiation (Fig. 1). Proliferating cells were compared with cells differentiating after withdrawal of FGF2. Cell lysates were prepared from proliferating stem cells (FGF2) and cortical stem cells at 1, 2, 4, and $6 \mathrm{~d}$ after FGF2 withdrawal (Fig. 1, No add.). Each cell lysate was first immunoprecipitated with antibodies to PDGF $\alpha$-receptor (R7), after which the supernatant was subjected to a second round of immunoprecipitation with anti-PDGF $\beta$-receptor antibodies (R33). The membranes were then hybridized with antibodies to the PDGF $\alpha$-receptor or the PDGF $\beta$-receptor. Anti-phosphotyrosine antibody was used to detect phosphorylation of the receptors. Figure 1 shows that 

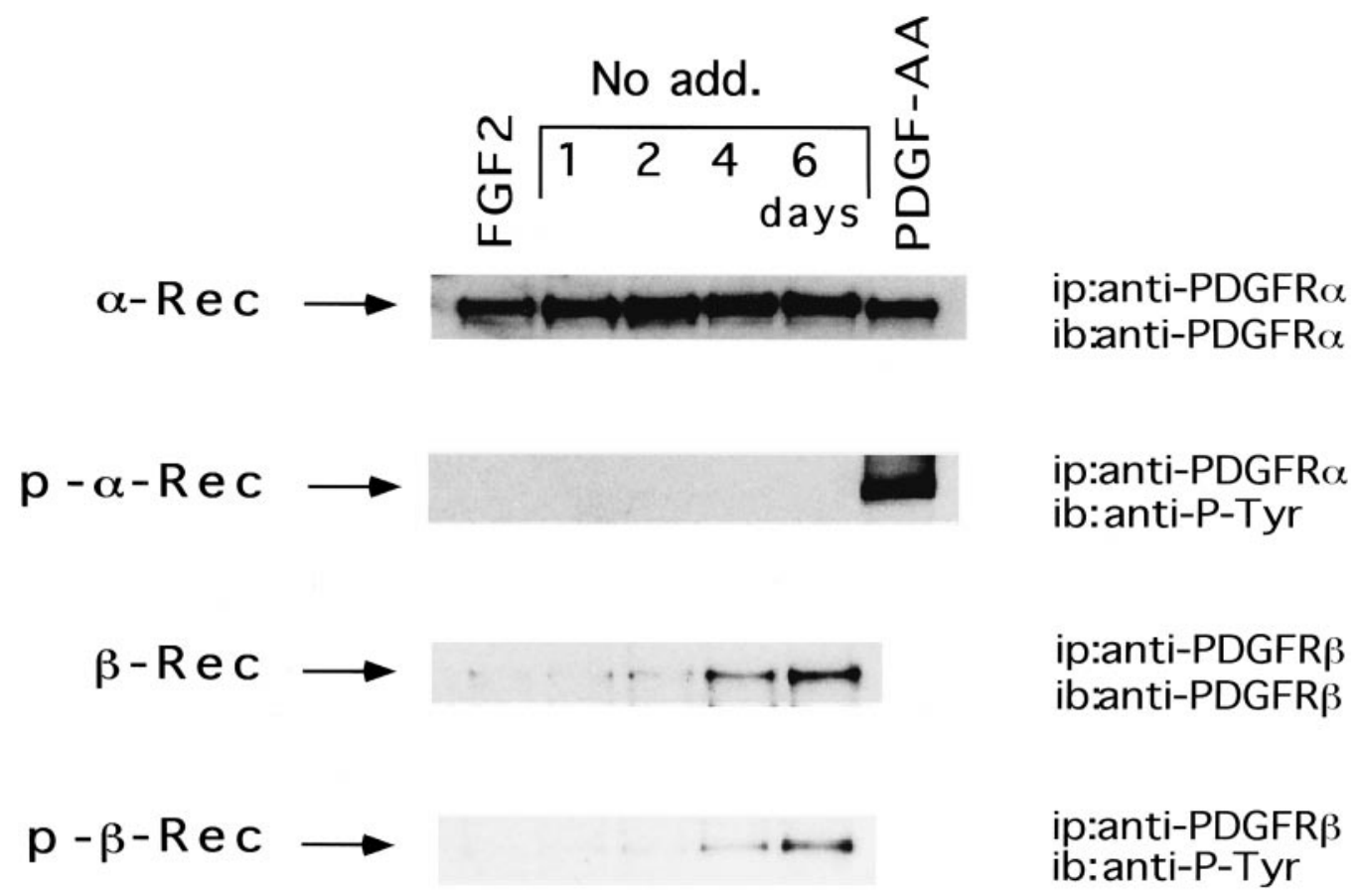

Figure 1. Western blot analysis was used to study PDGF receptor expression and phosphorylation in CNS stem cells during differentiation. Proliferating cells (FGF2) were compared with differentiating cells after FGF2 withdrawal (No add.). All cell lysates were immunoprecipitated with a PDGF $\alpha$-receptor antibody. For immunoprecipitation of the PDGF $\beta$-receptor, the supernatant from the $\alpha$-receptor precipitation was further incubated with a PDGF $\beta$-receptor antibody. Anti-PDGF $\alpha$-receptor antibody, anti-PDGF $\beta$-receptor antibody, or anti-phosphotyrosine antibody was used for immunoblotting. Lysates were made from cortical stem cells grown in the presence of FGF2, at the start of the experiment, and from cells grown 1, 2, 4, and $6 \mathrm{~d}$ after FGF2 withdrawal. Cells treated with PDGF-AA for $24 \mathrm{hr}$ were included as a positive control for phosphorylated PDGF $\alpha$-receptor.

although the PDGF $\alpha$-receptor is expressed on cortical stem cells, no phosphorylation occurred in the absence of exogenous ligand. Proliferating stem cells treated with $20 \mathrm{ng} / \mathrm{ml}$ of PDGF-AA were used as a positive control for phosphorylated $\alpha$-receptor.

PDGF $\beta$-receptor expression in cortical stem cells was hardly detectable in uncommitted cells but increased as the cells differentiated after FGF2 withdrawal. The phosphorylation of the $\beta$-receptor followed the same pattern, with no or very little phosphorylation the first $2 \mathrm{~d}$ of differentiation and a distinct phosphorylation at day 4 that increased further until day 6 after FGF2 withdrawal. On the basis of these and previous results from our laboratory we concluded that the PDGF $\alpha$-receptor is the predominant receptor isoform during early stem cell differentiation.

\section{Expression of PDGF receptors after PDGF-AA stimulation}

Because PDGF has been implicated in neuronal differentiation of CNS stem cells, we next investigated how receptor levels were influenced by differentiation in the presence of PDGF. Western blot analysis was used to study expression and phosphorylation of PDGF receptors in neuroepithelial cells after PDGF-AA stimulation. Subconfluent cortical stem cells were grown for 1, 2, 4, and $6 \mathrm{~d}$ after withdrawal of FGF2, in the presence of $10 \mathrm{ng} / \mathrm{ml}$ of PDGF-AA, and compared with proliferating stem cells (FGF2). Because previous investigators (Williams et al., 1997) suggest that a short exposure to PDGF suffices to stimulate neuronal differentiation, parallel cultures received a single dose of $20 \mathrm{ng} / \mathrm{ml}$ PDGF-AA on day 0, after which they were kept in plain N2-medium.

Figure $2 A$ shows that the PDGF $\alpha$-receptor expression level remained constant throughout the experiment, in which cortical stem cells were treated continuously with PDGF-AA. In cultures treated with a single dose of PDGF-AA, the PDGF $\alpha$-receptor expression level decreased $4 \mathrm{~d}$ after withdrawal of FGF2 (Fig. 2B).

After PDGF-AA stimulation, phosphorylation of the PDGF $\alpha$-receptor in cortical stem cell cultures reached a maximum the first day after stimulation. Thereafter, the level of receptor phosphorylation decreased but was maintained at a level higher than that of control cells (Fig. 2A). The phosphorylation level in cells that received a single dose of PDGF also decreased after a peak on day 1 and reached control levels at $4 \mathrm{~d}$ after stimulation (Fig. 2B).

Similarly to cortical stem cells grown in the absence of PDGF, the PDGF $\beta$-receptor level for PDGF-treated cells was very low at the beginning of the experiment, but the expression increased as the cells differentiated. In contrast to cultures differentiating in plain N2-medium, in which a distinct phosphorylation of the PDGF $\beta$-receptor was seen at days 4 and 6 , no phosphorylation of the $\beta$-receptor was detected in cultures treated with PDGF-AA (data not shown).

\section{PDGF induces c-fos RNA expression}

In our examination of cellular events initiated by activation of PDGF receptors, we next studied expression of the immediateearly gene c-fos in response to PDGF-AA. We first incubated cortical stem cell cultures for $3 \mathrm{hr}$ in N2-medium without FGF2 to minimize possible c-fos background expression. Cells were then stimulated with PDGF-AA and harvested for RNA preparation after $10 \mathrm{~min}, 30 \mathrm{~min}, 1 \mathrm{hr}$, and $2 \mathrm{hr}$. Control cells were incubated for $3 \mathrm{hr}$ without FGF, after which RNA was prepared. In Figure 3 the ratio of c-fos RNA to GAPDH RNA is shown. The PDGFAA-stimulated c-fos expression displayed a maximal induction of 


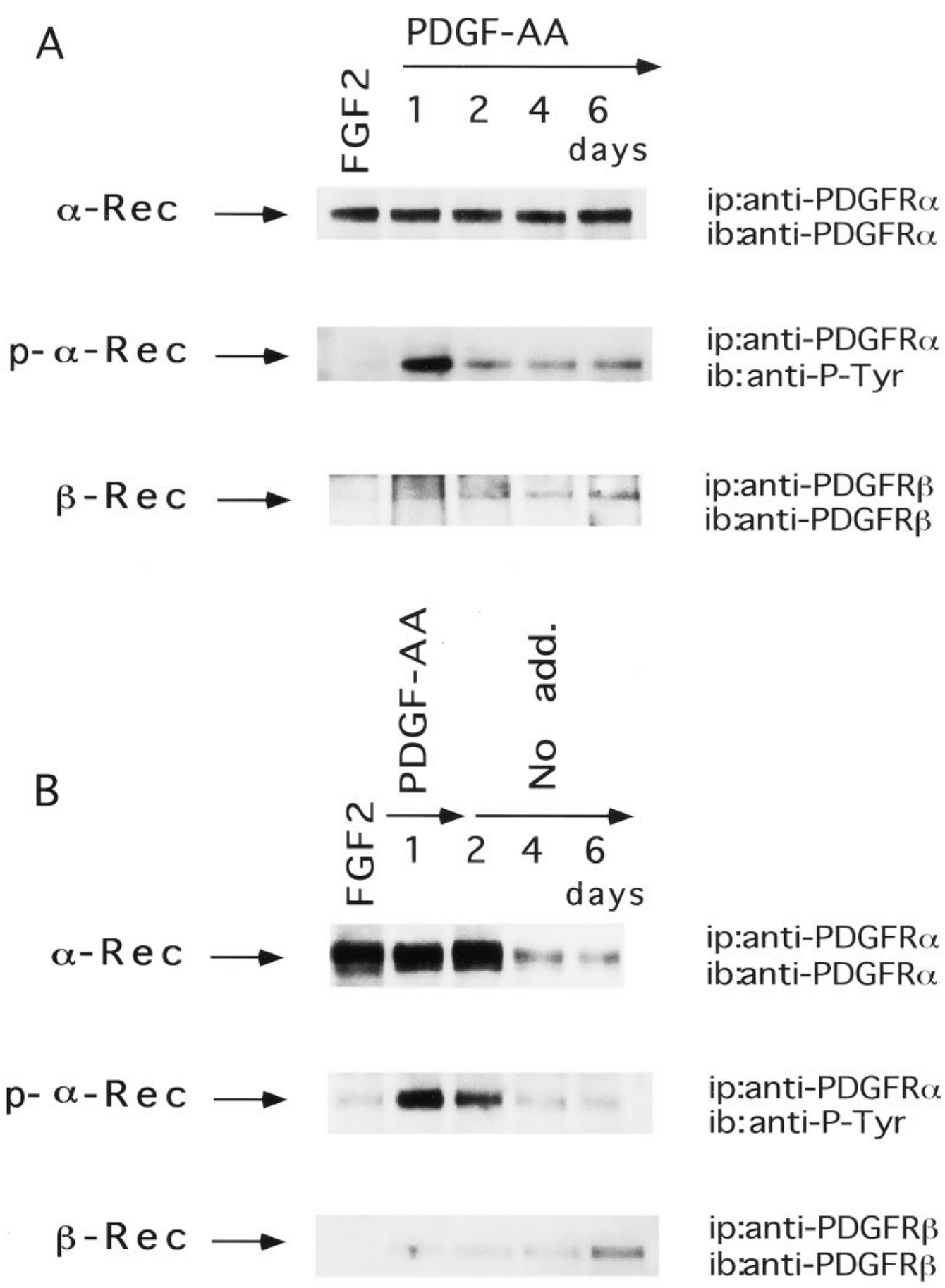

Figure 2. Western blot analysis of PDGF receptor expression and phosphorylation in CNS stem cells after PDGF-AA stimulation. Immunoprecipitation of cell lysates was performed using antibodies to PDGF $\alpha$-receptor and PDGF $\beta$-receptor. Anti-PDGF $\alpha$-receptor antibody, anti-PDGF $\beta$-receptor antibody, or anti-phosphotyrosine antibody was used for immunoblotting. Lysates were made from cortical stem cells grown in the presence of FGF2 (at the start of the experiment), from cells treated continuously with PDGF-AA after the withdrawal of FGF2 $(A)$, and from cells stimulated with a single dose of PDGF-AA at the time of FGF2 withdrawal $(B)$.

50-fold the control level at $1 \mathrm{hr}$. At $2 \mathrm{hr}$ the amount of c-fos RNA had declined but had not yet reached that of control cells.

\section{PDGF-AA stimulation of BrdU incorporation}

To investigate whether PDGF-AA can stimulate DNA synthesis in cortical stem cells, cultures were pulse labeled with BrdU for 14 hr and stained with an anti-BrdU antibody. The BrdU incorpo- ration in FGF2-cultured cells were $86 \pm 2.9 \%$ (data not shown). Cells grown in the absence of FGF2 were compared with cells treated once with PDGF-AA, at the time of FGF2 withdrawal, and cells treated continuously with PDGF-AA (Fig. 4). Counting of BrdU-positive cells revealed that treatment with PDGF-AA gives a fourfold increase in BrdU incorporation $2 \mathrm{~d}$ after stimulation, compared with control cultures that received no PDGF. In 


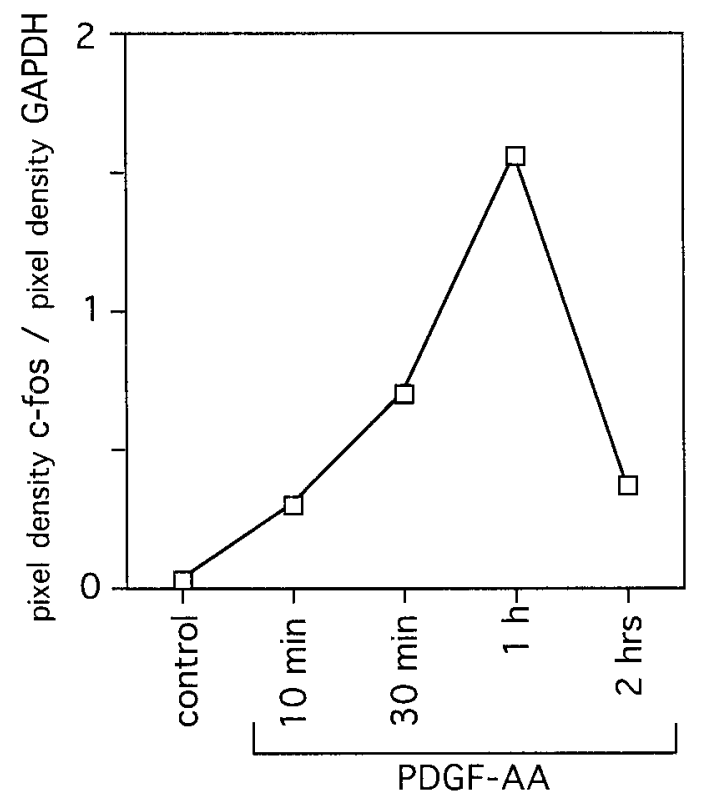

Figure 3. Northern blot analysis of c-fos expression in cortical stem cells after PDGF-AA stimulation. Cell cultures were incubated without FGF2 for $3 \mathrm{hr}$, stimulated with PDGF-AA, and harvested for RNA preparation after $10 \mathrm{~min}, 30 \mathrm{~min}, 1 \mathrm{hr}$, and $2 \mathrm{hr}$. The levels of RNA expression are given as the ratios of c-fos to GAPDH pixels.

cultures receiving a single dose of PDGF, the amount of cells incorporating BrdU had declined to that of control cells after $4 \mathrm{~d}$. In cells treated continuously with PDGF-AA, the number of BrdU-positive cells remained significantly higher after $4 \mathrm{~d}$. In all PDGF-treated cells, BrdU incorporation levels had declined to that of control cells after $6 \mathrm{~d}$.

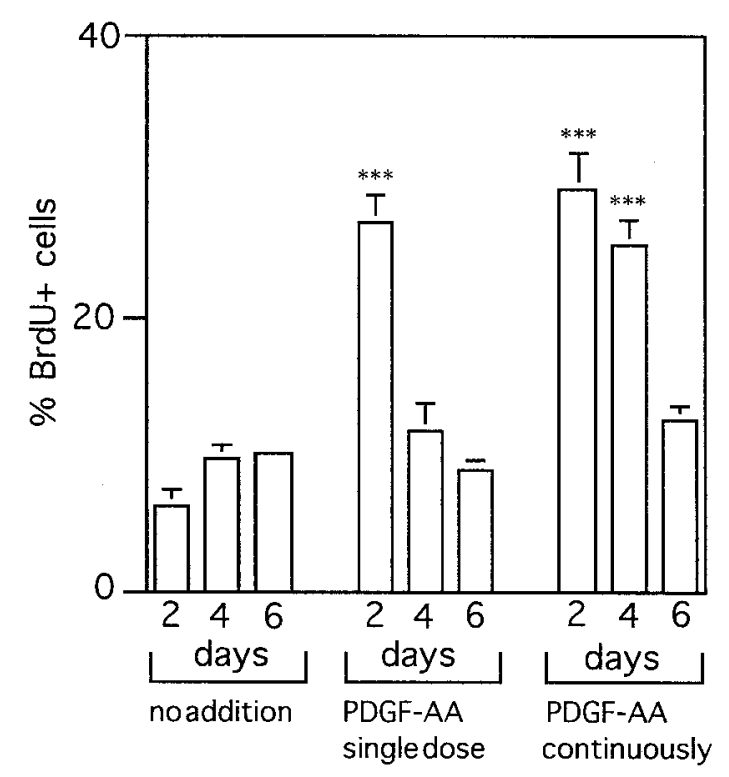

Figure 4. BrdU labeling of PDGF-AA-stimulated cortical stem cells. Parallel stem cell cultures were untreated, treated once with PDGF-AA, or treated continuously with PDGF-AA for 2, 4, and $6 \mathrm{~d}$. Before fixation the cells were exposed to BrdU for $14 \mathrm{hr}$. Incorporation of BrdU was detected using anti-BrdU antibodies. Stained cells (duplicate dishes) were counted (in seven parallel fields; $200 \times$ magnification) and plotted as the ratio of BrdU-positive cells to the total cell number. ${ }^{* * *}$ denotes $p$ $<0.001$.

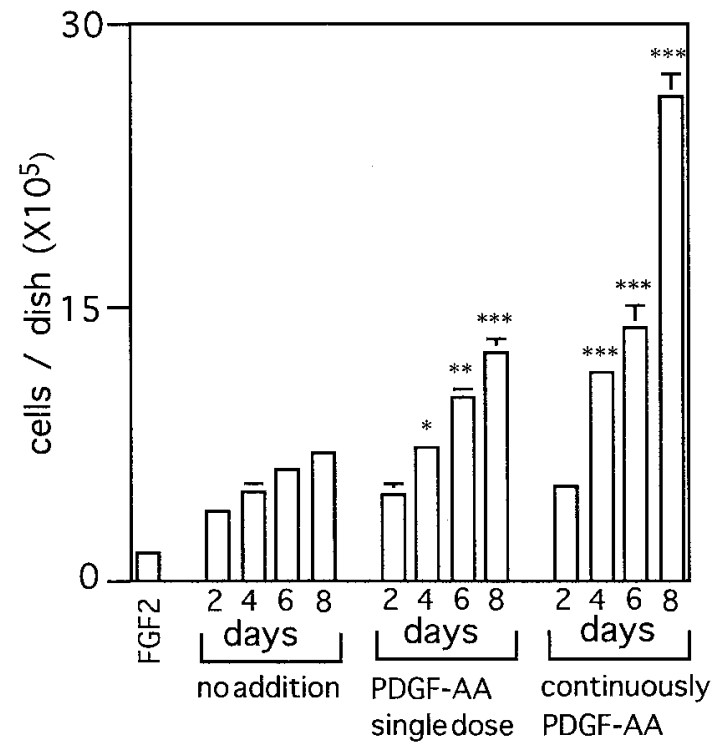

Figure 5. Total cell number in cortical cultures after PDGF-AA treatment. FGF2-treated cells were harvested and counted at the start of the experiment. After FGF2 withdrawal, parallel stem cell cultures were untreated, treated with a single dose of PDGF-AA, or treated continuously with PDGF-AA for 2, 4, 6, and 8 d. The total cell number was measured using a Coulter Z1 cell counter. * denotes $p<0.05,{ }^{* *} p<0.01$, and ${ }^{* * *} p<0.001$.

\section{PDGF-AA treatment increases the total cell number}

To further clarify the mitogenic effect of PDGF-AA on cortical stem cells, we performed cell counting experiments. FGF2treated cells were harvested and counted at the start of the experiment. After FGF2 withdrawal, cultures were left untreated, treated once with PDGF-AA, or treated continuously with PDGF-AA. The total cell number was measured using a Coulter Z1 cell counter, on days 2, 4, 6, and 8 (Fig. 5). A fourfold increase in total cell number was observed in cortical cultures treated continuously with PDGF-AA, compared with untreated cultures, at day 8 . The increase in total cell number was lower, but significant, in cultures treated with a single dose of PDGF-AA.

\section{PDGF treatment retains an immature phenotype that can be stained with neuronal markers}

To further investigate the effect of PDGF on cortical stem cell differentiation, cells were stained with antibodies specific to the neuronal marker, MAP2. Cells were grown for 2, 4, or $6 \mathrm{~d}$ without FGF2 in the absence or presence of PDGF. Parallel cultures were untreated, received a single dose of PDGF-AA, or received a daily dose of PDGF-AA (Fig. 6). After fixation, the cells were stained with MAP2 antibodies to reveal neuronal differentiation. Parallel cultures were stained with antibodies to nestin. At the time of FGF2 withdrawal, close to $100 \%$ of the cells were nestin positive, and this number declined during the course of the experiment, as the cells differentiated (data not shown). In cultures grown in the presence of FGF2, very few MAP2-positive cells were seen (Fig. 6A). In untreated cultures, MAP2-positive cells started to appear $2 \mathrm{~d}$ after FGF withdrawal, indicative of neuronal differentiation (Fig. $6 B$ ). The intensity of MAP2 staining in control cultures increased with time (Fig. $6 E, H$ ), and $6 \mathrm{~d}$ after FGF2 withdrawal $(H)$ most the cells had differentiated. The MAP2-positive neurons now had a more mature phenotype with extended processes (Fig. $6 H$ ).

Cells treated with a single dose of PDGF-AA showed the same 


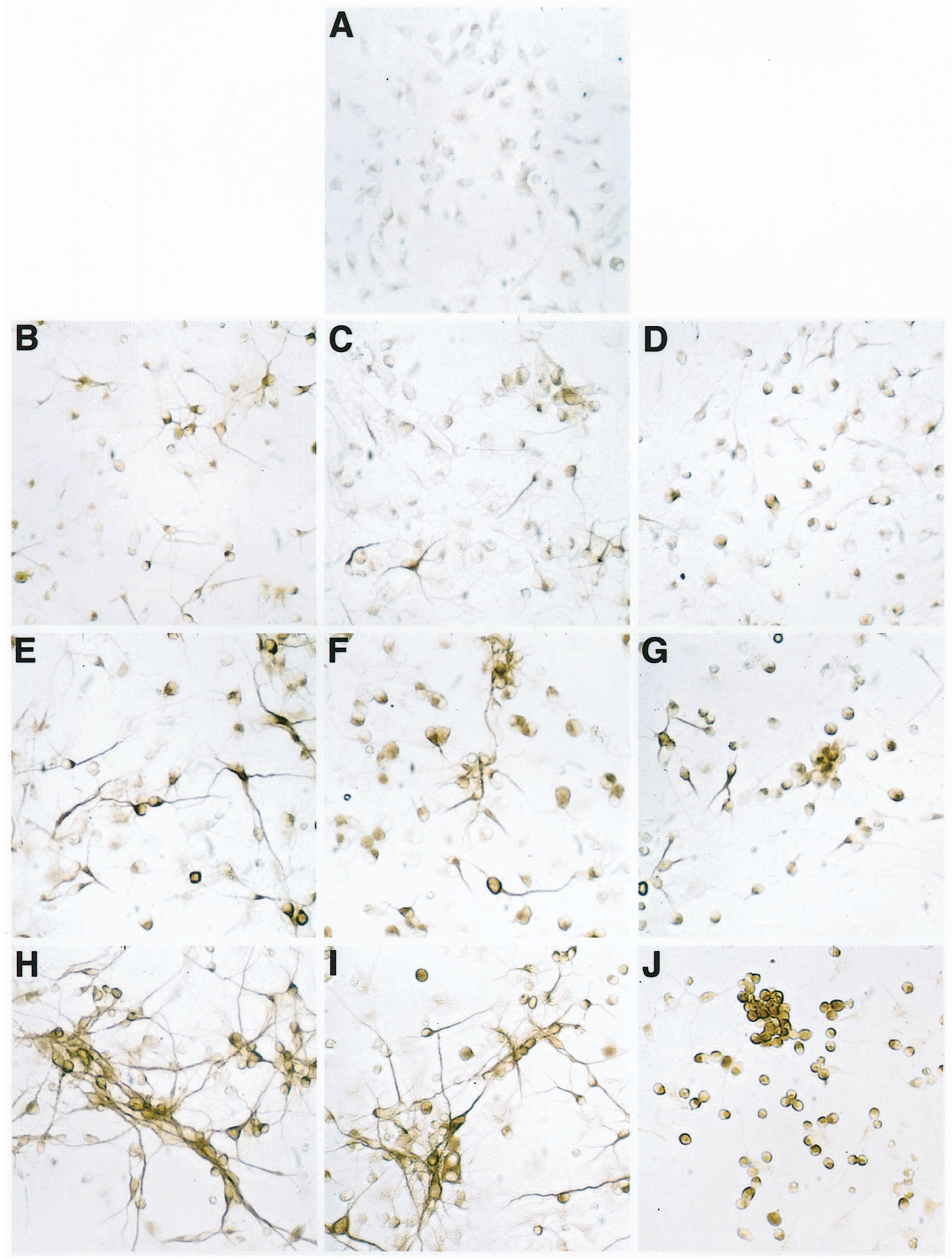

Figure 6. Staining of PDGF-AA-treated cortical stem cells with neuronal markers. After fixation, the cells were stained with MAP2 antibodies. Control cells, grown in the presence of FGF2, were fixed at the start of the experiment $(A)$. Cells were grown for $2 \mathrm{~d}(B-D), 4 \mathrm{~d}(E-G)$, or $6 \mathrm{~d}(H-J)$ without FGF2 in the absence or presence of PDGF. Parallel cultures were untreated $(B, E, H)$, received a single dose of PDGF-AA $(C, F, I)$, or PDGF-AA was added daily $(D, G, J)$. The cells were photographed at $200 \times$ magnification.

type of differentiation pattern (Fig. $6 C, F, I$ ), but the differentiation process was slightly slower, with more immature MAP2positive cells at days 2 and 4 than in the untreated cultures. In contrast, the MAP2-positive cells in cultures treated continuously with PDGF-AA did not differentiate into process-extending neurons (Fig. $6 D, G, J$ ) during the course of $6 \mathrm{~d}$. Still, at the end of the experiment $(J)$ only a few cells showed morphological maturation. The MAP2-positive cells rather display a rounded morphol- 


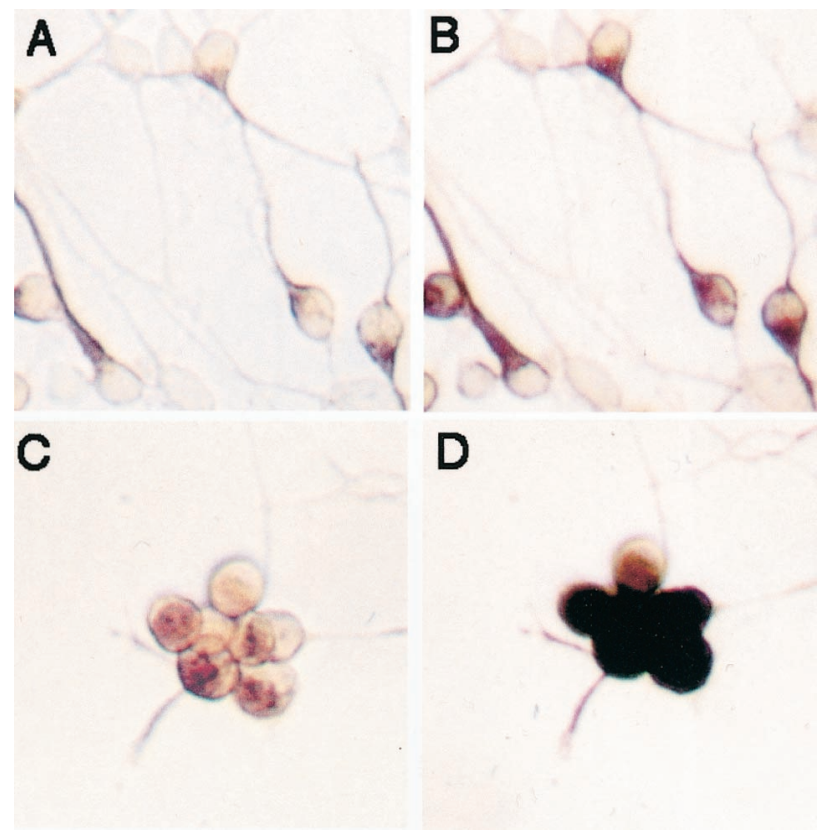

Figure 7. MAP2 and BrdU double staining of PDGF-AA-treated cortical cells. Stem cell cultures were untreated $(A, B)$ or treated with PDGF-AA $(C, D)$ for $2 \mathrm{~d}$ after FGF2 withdrawal. The cells were exposed to $\mathrm{BrdU}$ for $14 \mathrm{hr}$, fixed, and stained with MAP2 antibodies $(A, C)$. Defined fields of the culture dish were photographed using a computerized microscope. The cultures were subsequently stained for BrdU, and the same fields were photographed again $(B, D)$.

ogy, and many cells appear in clusters. Together with the data from the BrdU incorporation and cell counting experiments, these results manifest a mitogenic effect of PDGF-AA on cortical stem cells during the early stage of neuronal differentiation. Parallel experiments were stained with an antibody to Tuj1, another commonly used neuronal marker, and an identical staining pattern was observed (data not shown).

\section{MAP2-positive progenitor cells incorporate BrdU in PDGF-AA-treated cultures}

To further investigate whether dividing cells are neuronal precursors, we used double staining of cortical stem cells with antibodies to MAP2 and BrdU (Fig. 7). Cells were grown for 2 or $4 \mathrm{~d}$ without FGF2 in the absence or presence of $10 \mathrm{ng} / \mathrm{ml}$ PDGF-AA. Before fixation, the cells were pulse labeled with BrdU for $14 \mathrm{hr}$. The cells were first stained for MAP2 and photographed, using a computerized microscope. After subsequent staining with BrdU antibodies, the same fields of the culture dish were photographed again. A high proportion of cells grown in the absence of PDGF for $2 \mathrm{~d}$ were MAP2 positive (Fig. 7A) and BrdU negative (Fig. 7B, Table 1) and extended short processes. Cells grown in the presence of PDGF-AA showed an undifferentiated phenotype $2 \mathrm{~d}$ after FGF2 withdrawal (Fig. 7C). Staining with antibodies to BrdU indicated that a population of the cells treated with PDGF-AA for $2 \mathrm{~d}$ were positive for both MAP2 and BrdU (Fig. $7 D$, Table 1). After $4 \mathrm{~d}$ of differentiation, the PDGF-treated cells still showed an immature phenotype, but the amount of doublepositive cells had decreased to control levels (Table 1). Untreated cells had a more differentiated phenotype with long processes (data not shown).

\section{PDGF-AA is involved in control of stem cell survival}

We next investigated whether the increase in cell number, seen after addition of PDGF-AA, results from increased cell survival
Table 1. Percentage of MAP2 and BrdU double-positive cells

\begin{tabular}{llc} 
& No addition & PDGF-AA \\
\hline Day 2 & $3.0 \pm 0.7 \%$ & $21.8 \pm 1.2 \%^{* * *}$ \\
Day 4 & $4.7 \pm 1.0 \%$ & $5.3 \% \pm 0.6 \%$
\end{tabular}

Cortical cultures were untreated (No addition) or treated with PDGF-AA for 2 and $4 \mathrm{~d}$. Cells were exposed to BrdU for $14 \mathrm{hr}$ before fixation and stained with MAP2 antibodies, and defined fields of the culture dish were photographed. After subsequent staining for BrdU, the same fields were photographed again. Ten parallel fields were counted for each time point, and the ratio of double-positive cells to the total cell number was calculated. $* * *$ denotes $p<0.001$.

in addition to the effect on proliferation. Staining for apoptotic nuclei using a TUNEL assay revealed no differences between PDGF-AA and untreated cultures at $2 \mathrm{~d}$ of differentiation, but at day 4 we detected a twofold decrease in TUNEL staining in PDGF-AA-treated cultures (Fig. $8 A$ ).

The serine threonine kinase PKB/c-Akt has been linked to increased cell survival. In addition to the TUNEL assay, we studied the phosphorylation of $\mathrm{PKB} / \mathrm{c}-\mathrm{Akt}$ as a measure of increased cell survival. Proliferating stem cell cultures were stimulated for 5 min with $100 \mathrm{ng} / \mathrm{ml}$ PDGF-AA or $100 \mathrm{ng} / \mathrm{ml} \mathrm{FGF2,}$ and total protein lysates were performed. Cell lysates from parallel cultures, receiving only medium, were used as a control. Figure $8 B$ shows that PDGF-AA stimulated a moderate PKB/cAkt phosphorylation. FGF2 treatment also resulted in stimulation of $\mathrm{PKB} / \mathrm{c}-\mathrm{Akt}$ phosphorylation, but the level was lower than in PDGF-treated cultures. No phosphorylated PKB/c-Akt was detected in untreated control cells. Total PKB/c-Akt levels were not found to be affected by PDGF-AA or FGF2 stimulation.

\section{DISCUSSION}

Multipotent neural stem cells can be maintained in monolayer cultures in the presence of FGF2, and on withdrawal of the mitogen give rise to neurons, astrocytes, and oligodendrocytes (for review, see McKay, 1997). The experiments described in this study were performed on stem cell cultures from the E15 rat cortex in their first passage. This provides a culture system with homogeneous cells, with respect to morphological criteria and staining for the intermediate filament nestin.

The influence of exogenous factors on differentiation of CNS stem cells has been addressed in several studies. Although the pathway of astrocytic differentiation induced by CNTF has been studied in detail (Bonni et al., 1997; Rajan and McKay, 1998), less is known about the development of the neuronal lineage in stem cells. Two reports (Johe et al., 1996; Williams et al., 1997) have suggested that PDGF-treatment of cortical progenitor cells leads to an increase in the number of neurons. In the study by Williams et al. (1997), a different cell culture protocol was used, and cortical cells were treated with PDGF-AA without previous expansion of stem cells. Therefore, their data cannot be directly compared with our results. The present investigation uses the method described in Johe et al. (1996). These authors raise the possibility that PDGF does not act as an instructive factor like CNTF, a statement that fits with our data.

PDGF is active either as a homodimer (AA, BB) or heterodimer $(\mathrm{AB})$. Activation of PDGF receptors is brought about by ligand binding to dimerizing receptor subunits $\alpha$ or $\beta$. The BB homodimer can only bind to $\beta$-receptor subunits, whereas PDGF-AA can activate both $\alpha$ - and $\beta$-receptors (Westermark and Sorg, 1993). The AA homodimer, however, has a slightly lower affinity for the $\beta$-receptor. In accordance with our previous 


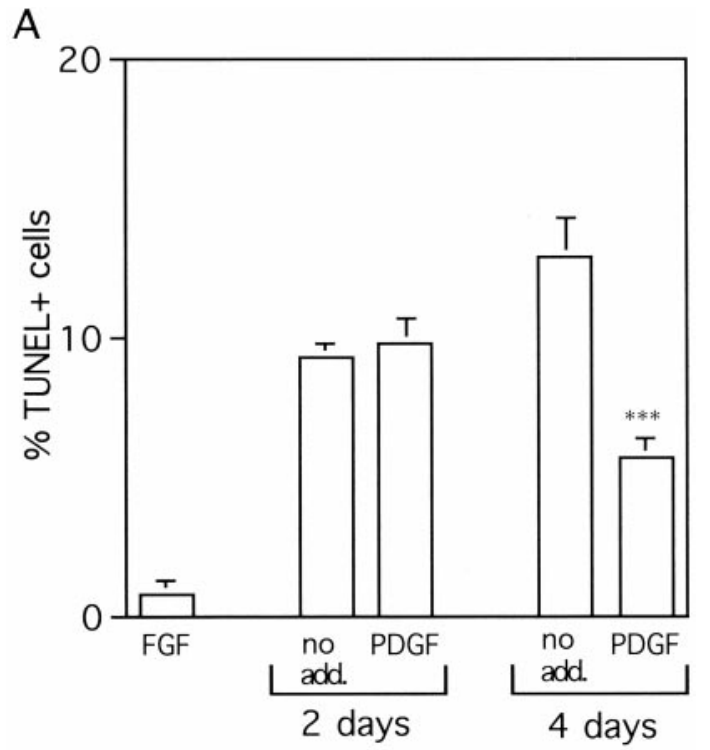

B
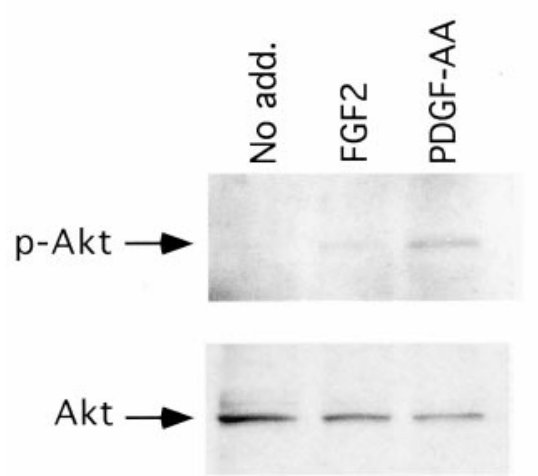

Figure 8. Cell survival studies of PDGF-AA-treated cortical stem cells. A TUNEL assay was performed on cells grown for 2 and $4 \mathrm{~d}$ in the presence or absence (no addition, no add.) of PDGF-AA. Cells grown in the presence of FGF2 were included as a control. Cells going through apoptosis were counted using a fluorescence microscope and plotted as the ratio to total number of cells $(A)$.*** denotes $p<0.001$. Western blot analysis was used to study $\mathrm{PKB} / \mathrm{c}-\mathrm{Akt}$ phosphorylation in CNS stem cells after FGF2 and PDGF-AA stimulation compared with untreated cells (no $a d d$.) (B). Total cell lysates were used for immunoblotting with anti-PKB/ c-Akt antibody (Akt) and anti-phosphorylated PKB/c-Akt antibody $(p-A k t)$.

data, we detected PDGF $\alpha$-receptor expression already on dissociated cortices at E15. In contrast, the $\beta$-receptors were hardly detected in uncommitted cells, suggesting that in cortical stem cells the $\alpha$-receptor is the predominant receptor isoform. Therefore we chose to use PDGF-AA as the ligand throughout this study.

Tyrosine phosphorylation of the PDGF $\alpha$-receptor was not seen in dissociated cortices, in analogy to previous investigations (Williams et al., 1997), and during differentiation, induced by discontinued addition of FGF2, we could not detect PDGF $\alpha$-receptor phosphorylation using phosphotyrosine antibodies. The cortical stem cells responded to addition of PDGF-AA by receptor phosphorylation, such that a prolonged exposure to the ligand gave rise to a sustained receptor activation. This prolonged tyrosine phosphorylation, however, never reached the level seen during the first day after stimulation, suggesting receptor downregulation in response to the ligand. In lysates prepared from cells that were given a single dose of PDGF, phosphorylation of the $\alpha$-receptor returned to background levels more quickly than in cells exposed continuously to PDGF-AA.

In contrast to the PDGF $\alpha$-receptor, the $\beta$-receptor is barely detectable during the first days of differentiation in cortical stem cells, and we could see no phosphorylation on tyrosine residues in response to PDGF-AA. Therefore, we conclude that the increased BrdU uptake and total cell number after PDGF-AA stimulation are mediated through PDGF $\alpha$-receptors. During differentiation induced by withdrawing FGF2, $\beta$-receptor expression can be visualized by Western blot analysis. Phosphorylation of the PDGF $\beta$-receptor was also seen when cortical stem cells differentiated in the absence of PDGF-AA. Immunohistochemical analysis of rat brain has shown that the PDGF $\beta$-receptor is expressed in neurons in many areas of the CNS (for review, see Valenzuela et al., 1997), and it has been reported that PDGF-BB exerts neurotrophic activity on neurons from newborn rats (Smits et al., 1991). Additional studies support the notion that PDGF-BB and the $\beta$-receptor are involved in survival and proliferation of neurons (Giacobini et al., 1993; Nikkhah et al., 1993). In view of these findings it is likely that the $\beta$-receptor, detected after several days of differentiation, reflects expression on neurons. However, the exact stage of maturation at which the $\beta$-receptor starts to be expressed remains to be determined.

Expression of immediate-early genes is induced in various tissues in response to a wide range of extracellular cues, for example, PDGF (Cochran and Weissman, 1984; Greenberg and Ziff, 1984; Kruijer et al., 1984; Müller et al., 1984). Among the immediate-early genes are common transcription factors, but also genes that are more restricted to differentiation of a specific cell lineage (for review, see Herschman, 1991). Core transcription factors include c-Myc and c-Fos, both of which are activated by PDGF in many cell types. PDGF-AA-stimulated c-fos RNA expression in cortical stem cells peaked at $1 \mathrm{hr}$, suggesting that it is mediated by signaling through PDGF $\alpha$-receptors. The possibility that c-fos activation is a secondary phenomenon is less likely, because of the time frame of induction.

From MAP2 (and Tuj1) staining of differentiating cortical cultures, it is evident that exogenous PDGF has a profound effect on cell morphology. The presence of PDGF-AA after withdrawal of FGF2 almost completely abolished morphological neuronal maturation. Instead of extending elaborate processes as in control cultures, PDGF-AA-treated cells retained a rounded immature phenotype even after $6 \mathrm{~d}$. It is interesting to note that a single dose of PDGF, at the time of FGF2 withdrawal, is not sufficient to obtain the same effect on differentiation. Cell culture medium is changed every second day, and any residual PDGF is washed out from the culture at that time. Thus, the presence of PDGF over several days of differentiation is needed for the effect reported in this investigation.

The immature morphology of PDGF-AA-treated stem cell cultures could be caused by a block in differentiation, a continuous proliferation, or an increase in cell survival, or a combination of these. Previous investigators (Johe et al., 1996) and our unpublished observations have shown that PDGF cannot substitute FGF2 as a major mitogen for cortical stem cells. When FGF2 is not present, however, our data from BrdU incorporation and cell counting experiments show that PDGF is able to stimulate divi- 
sion of stem cells. Both a single dose and renewed addition of PDGF-AA gave a fourfold increase in BrdU-positive cells at $2 \mathrm{~d}$, whereas at $4 \mathrm{~d}$ a continuous presence of PDGF was needed to yield a higher percentage compared with control cells. Double staining for MAP2 and BrdU reveals a population of cells that express a neuronal marker while still dividing.

The possible effect of PDGF-AA on cell survival was investigated. TUNEL staining revealed that the amount of cells going through apoptosis during the earliest phase of differentiation (2 d) is similar between control and PDGF-treated cultures. At $4 \mathrm{~d}$ of differentiation, however, the number of apoptotic nuclei was reduced by half in the presence of PDGF. Activation of $\mathrm{PKB} / \mathrm{c}-$ Akt has been linked to reduced apoptosis (Kandel and Hay, 1999) and was shown to act as a survival factor for cerebellar neurons (Dudek et al., 1997). A modest phosphorylation of PKB/ c-Akt was also seen in response to PDGF-AA. Thus, in addition to stimulating stem cell proliferation, PDGF also exerts a cell survival effect.

Our results fit with the notion that PDGF stimulates a neuronal fate of stem cells, although not as an instructive agent but rather to expand a pool of immature neurons before their morphological maturation occurs.

PDGF-AA and the $\alpha$-receptor play an important role in the development of the oligodendrocyte lineage. A recent report points at an unexpected plasticity of early oligodendrocyte progenitors (Kondo and Raff, 2000). To evaluate the differentiation capacity of the $\mathrm{MAP}^{2+} / \mathrm{BrdU}+$ progenitors in this study on treatment with agents that promote oligodendrocyte development, such as T3, is therefore greatly warranted.

\section{REFERENCES}

Auffrey C, Rougeon F (1980) Purification of mouse immunoglobulin heavy-chain messenger RNAs from total myeloma tumor RNA. Eur J Biochem 107:303-314.

Bonni A, Sun Y, Nadal-Vicens M, Bhatt A, Frank D, Rozovsky I, Stahl N, Yancopoulos G, Greenberg M (1997) Regulation of gliogenesis in the central nervous system by the JAK-STAT signaling pathway. Science 278:477-483.

Bottenstein JE, Sato GH (1979) Growth of a rat neuroblastoma cell line in a serum-free supplemented medium. Proc Natl Acad Sci USA 76:514-517.

Cochran MD, Weissman C (1984) Molecular structure of b-globin and the TK promoters. EMBO J 3:2453-2459.

Dudek H, Datta SR, Franke TF, Birnbaum MJ, Yao R, Cooper GM, Segal RA, Kaplan DR, Greenberg ME (1997) Regulation of neural survival by the serine-threonine protein kinase Akt. Science $275: 661-665$.

Forsberg-Nilsson K, Behar T, Afrakhte M, Barker J, McKay RDG (1998) Platelet-derived growth factor induces chemotaxis of neuroepithelial stem cells. J Neurosci Res 53:521-530.
Gage FH (1998) Stem cells of the central nervous system. Curr Opin Neurobiol 8:671-676.

Giacobini MM, Almstrom S, Funa K, Olson L (1993) Differential effects of platelet-derived growth factor isoforms on dopamine neurons in vivo: -BB supports cell survival, -AA enhances fiber formation. Neuroscience 57:923-929.

Greenberg ME, Ziff EB (1984) Stimulation of 3T3 cells induces transcription of the c-fos proto-oncogene. Nature 312:656-658.

Hatten ME (1990) Riding the glial monorail: a common mechanism for glial-guided migration in different regions of the developing brain. Trends Neurosci 13:179-184.

Herschman HR (1991) Primary response genes induced by growth factors and tumor promoters. Annu Rev Biochem 60:281-319.

Hutchins J, Jefferson V (1992) Developmental distribution of plateletderived growth factor in the mouse central nervous system. Dev Brain Res 67:121-135.

Johe KK, Hazel TG, Muller T, Dugich Djordjevic MM, McKay RDG (1996) Single factors direct the differentiation of stem cells from the fetal and adult central nervous system. Genes Dev 10:3129-3140.

Kandel ES, Hay N (1999) The regulation and activities of the multifunctional serine/threonine kinase Akt/PKB. Exp Cell Res 253:210-229.

Kondo T, Raff M (2000) Oligodendrocyte precursor cells reprogrammed to become multipotential CNS stem cells. Science 289:1754-1757.

Kruijer W, Cooper JA, Hunter T, Verma IM (1984) Platelet-derived growth factor induces rapid but transient expression of the c-fos gene and protein. Nature 312:711-716.

McKay RDG (1997) Stem cells in the central nervous system. Science 276:66-71.

Müller R, Bravo R, Burckhardt J, Curran T (1984) Induction of c-fos gene and protein by growth factors precedes activation of c-myc. Nature $312: 716-720$

Nikkhah G, Odin P, Smits A, Tingstrom A, Othberg A, Brundin P, Funa K, Lindvall O (1993) Platelet-derived growth factor promotes survival of rat and human mesencephalic dopaminergic neurons in culture. Exp Brain Res 92:516-523.

Rajan P, McKay RDG (1998) Multiple routes to astrocytic differentiation in the CNS. J Neurosci 18:3620-3629.

Rakic P (1972) Mode of cell migration of the superficial layers of fetal monkey neocortex. J Comp Neurol 145:61-84.

Rakic P (1990) Principles of neural cell migration. Experientia 46:880-891.

Sasahara A, Kott J, Sasahara M, Raines E, Ross R, Westrum L (1992) Platelet-derived growth factor B-chain-like immunoreactivity in the developing and adult brain. Dev Brain Res 68:41-53.

Smits A, Kato M, Westermark B, Nistér M, Heldin C-H, Funa K (1991) Neurotrophic activity of platelet-derived growth factor (PDGF): rat neuronal cells possess functional PDGF $\beta$-type receptors and respond to PDGF. Proc Natl Acad Sci USA 88:8159-8161.

Temple S, Alvarez-Buylla A (1999) Stem cells in the adult mammalian nervous system. Curr Opin Neurobiol 9:135-141.

Valenzuela CF, Kazlauskas A, Weiner JL (1997) Roles of plateletderived growth factor in the developing and mature nervous systems. Brain Res Rev 24:77-89.

Vescovi AL, Snyder EY (1999) Establishment and properties of neural stem cell clones: plasticity in vitro and in vivo. Brain Pathol 9:569-598.

Westermark B, Sorg C (1993) Cytokines (Sorg C, ed). In: Biology of platelet-derived growth factor. Basel: Karger.

Williams B, Read J, Price J (1991) The generation of neurons and glia from a common precursor cell. Neuron 7:685-693.

Williams B, Park J, Alberta J, Muhlebach S, Hwang G, Roberts T, Stiles C (1997) A PDGF-regulated immediate early gene response initiates neuronal differentiation in ventricular zone progenitor cells. Neuron 18:553-562. 